\title{
ON MULTIPLE INTEGRALS
}

\author{
BY

\section{JAMES PIERPONT}

\section{§1. Introduction.}

In the theory of nultiple proper integrals the chief difficulty arises in the consideration of the frontier points of the field of integration $\mathfrak{A}$. The older writers as Gauss, Dirichlet, JACOBI and CAUCHY, supposed the field $\mathfrak{A}$ to be bounded by a finite number of surfaces of simple nature. In later years the theory of point aggregates has made it possible to consider more general classes of fields. Stolz, Pringsheim $\dagger$ and Jordan $\ddagger$ have made valuable contributions in this direction. The work of JORDAN is particularly important as he has not only blocked out the way for multiple integrals in any number of variables but has treated the case of two variables with great generality and completeness.

Two fundamental theorems in this theory relate to the reduction of a multiple integral to an iterated integral and the change of variables in a multiple integral. As far as the writer was aware a year ago when he began to consider the general treatment of these questions, none of the existing methods admitted an extension to $n$ variables, at least not without considerable complication. It occurred to him that by properly generalizing the definition of an integral the grave difficulties which the frontier points create might very readily be obviated.

The theory of integration which is here given, was presented by the author at the April.meeting of the American Mathematical Society in 1904. At that time Professor Moore called his attention to a paper of STOLz's, $\$$ published in 1897, where not only the integrals considered in the present paper but also another class of integrals are introduced. The importance of the integrals first mentioned seems entirely to have escaped Stolz's observation. At any rate he seems to have made no further use of them, and to have considered them more as curiosities. The only theorem he establishes is theorem 2 of the present paper.

* Presented to the Society, A pril 30, 1904. Received for publication May 19, 1905.

†Sitzungsberichte der M ii uchener Akademie, vol. 29 (1899), p. 39.

† Journal de Mathematiques, ser. 4, vol. 8 (1892), p. 69, and Cours d'Analyse, vol. 1.

₹ Zwei Grenzuerthe, von welchen das obere Integral ein besonderer Full ist, Sit zu ngsberich te ter Wiener A kademie, vol. 106, abt. II, $a$, p. 453. 


\section{\$2. Preliminary definitions.}

It is convenient to represent the points $x=\left(x_{1}, \cdots, x_{m}\right)$ of an $m$-way space $l_{m}$ by a set of points marked off on $m$ axes. Let us divide each of these axes into intervals of length $\leqq d$; in such a manner, however, that no finite segment of any axis contains more than a finite number of intervals. We shall say that we have effected a rectangular division of space of norm $d$. It divides $R_{m}$ into rectangular cells whose sides are of length $\leqq l$. The boundaries of the cells are planes parallel to the coördinate-planes. When the lengths of the intervals are all the same, these cells become cubes. The corresponding division may be called cubicul.

Let $\mathfrak{H}$ be a linited point aggregate, $i$. e., let no coördinate of any point of $\mathfrak{A}$ be greater numerically than some fixed number.

Let $f\left(x_{1}, \cdots, x_{m}\right)$ be a limited one-valued function defined over $\mathfrak{A}$. Let $D$ be a rectangular division of space of norm $d$. Let $d_{1}, d_{2}, d_{3}, \ldots$ denote those cells of $I$ containing at least one point of $\mathscr{U}$. Without ambiguity we may denote the volume of these cells by the same letters.

Let

and

Wre shall set

$$
M_{i}=\max , t, \quad m_{i}=\min f, \quad \text { in } d_{i} ;
$$

$$
\left|f\left(x_{1}, \ldots, x_{m}\right)\right| \leqq F, \quad \text { in } \mathscr{H} \text {. }
$$

$$
\bar{S}_{D}=\sum_{i} M_{i} d_{i}, \quad \underline{S}_{D}=\sum_{i} m_{i} d_{i}
$$

which may be called the "Ipper and lower sums for the division $D$. It is sometimes necessary to consider such sums over different aggregates as $\mathfrak{A}, \mathfrak{B}, \ldots$ These may be indicated by

$$
\bar{S}_{n_{D}}, \bar{S}_{*_{1} D}, \cdots,
$$

where the subscripts indicate that the sums (1) are taken over $\mathfrak{A}, \mathscr{H}, \ldots$ respectively.

The difference $M I_{i}-m_{i}$ is the oscillation of $f$ in the cell $l_{i}$. We denote it by

$$
\text { osc } f_{i}^{\prime} \text {. }
$$

We remark here once for all that all functions and point aggregates considered in this paper are supposed to be limited. The case when either or both are unlimited will be considered in a later paper.

Let $a=\left(a_{1}, \cdots, a_{m}\right), b=\left(b_{1}, \cdots, b_{m}\right)$ be two points. The distance between $a$ and $b$ is defined by

$$
\operatorname{dist}(\iota, b)=1^{\prime}\left(a_{1}-b_{1}\right)^{2}+\cdots+\left(a_{m}-b_{m}\right)^{2} .
$$


If $c$ is any other point, we have

$$
\operatorname{dist}(a, c) \leqq \operatorname{dist}(a, b)+\operatorname{dist}(b, c) .
$$

Let $\mathfrak{Z}$ be a partial aggregate of $\mathfrak{A}$. If the distance between any frontier point $\mathfrak{A}$ and any frontier point of $\mathfrak{B}$ is $\geqq \rho>0$, we say $\mathfrak{B}$ is an inner aggregate of $\mathfrak{A}$; also $\mathfrak{A}$ is an outer aggregate of $\mathfrak{B}$.

If $\mathfrak{A}$ contains all its limiting points, it is complete. If it is complete and contains only limiting points, it is perfect.

A point aggregate embracing none of its frontier points is called a region.

The points $x$ such that dist $(a, x) \leqq \delta$ form the domain of $a$ of norm $\delta$. We denote it by $D_{\delta}(a)$.

\section{§3. Fundamental theorems.}

TheOREM 1. Let $f\left(x_{1}, \cdots, x_{m}\right) \geqq 0$ be defined over the field, $\mathfrak{A}$. Let

$$
\bar{S}=\min S_{D}
$$

with respect to all rectangular division $D$ of norm $d$, however small. Then

$$
\lim _{d=0} \bar{S}_{D}=\bar{S} \text {. }
$$

The points of $\mathfrak{A}$ lie in a certain cube $\mathfrak{C}$ of edge $C$. The representation of $\mathfrak{C}$ is formed of $m$ segments $\mathfrak{C}_{1}, \ldots, \mathfrak{C}_{m}$ on the $x_{1}, \ldots, x_{m}$ axes. We may suppose $\mathfrak{C}$ taken so large that no point of $\mathfrak{A}$ comes within a certain distance of any side of $\mathfrak{E}$.

Since $\bar{S}$ is obviously finite, there exists for each $\epsilon>0$ a division $\Delta$ such that

$$
\bar{S} \leqq \bar{S}_{\Delta}<\bar{S}+\underset{2}{\epsilon}
$$

Let $D$ be an arbitrary division of norm $d$. Let us superimpose $\Delta$ on $D$, forming a division $E$. The division $E$ is formed by interpolating certain points, let us say at most $\mu$ in each of the segments $\mathfrak{E}_{1}, \ldots, \mathfrak{C}_{m}$. The interpolation of one of these points may be interpreted as passing a plane parallel to one of the sides of $\mathfrak{C}$. Its effect is to subdivide certain of the cells of $\mathfrak{C}$. The volume of the cells so effected is

$$
\leqq d C^{m-1}
$$

Hence the superimposition of $\Delta$ on $D$, being equivalent to passing at most $m \mu$ planes, affects cells of $\mathfrak{C}$ belonging to the original division $D$, whose volume is

$$
V<m \mu d C^{m-1} \text {. }
$$


Let $\Delta$ subdivide $d_{i}$, a cell of $D$ containing points of $\mathfrak{A}$, into the cells

$$
d_{i 1}, d_{i 2}, \cdots
$$

containing points of $\mathfrak{A}$ and into cells

$$
\delta_{i 1}, \delta_{i 2}, \cdots
$$

containing no points of $\mathfrak{A}$. Then

$$
\bar{S}_{D}=\sum_{i} M_{i} d_{i}+R ; \quad \bar{S}_{k}=\sum_{i k} M_{i k} d_{i k}+R,
$$

where $R$ denotes the sum of those terms common to $\bar{S}_{D}$ and $\bar{S}_{E}$ corresponding to cells of $D$ unaffected by the division $\Delta$. But

bence

$$
d_{i}=\sum_{i k} d_{i k}+\sum_{i k} \delta_{i k}
$$

Thus

$$
\bar{S}_{D}=\sum M_{i} d_{i k}+\sum M_{i} \delta_{i k}+R \text {. }
$$

$$
\begin{gathered}
0 \leqq \bar{S}_{D}-\bar{S}_{E} \leqq \sum\left(M_{i}-M_{i k}\right) d_{i k}+\sum M_{i} \delta_{i k} \\
<F \sum d_{i k}+F \sum \delta_{i k}=F V
\end{gathered}
$$

by (3). If we take

$$
<m \mu d F C^{m-1} \text {, }
$$

we have

$$
d^{\prime}<\frac{\epsilon}{2 m \mu \bar{F} C^{m-1}}
$$

$$
\bar{S}_{D}<\bar{S}_{E}+\frac{\epsilon}{2}
$$

for any $d \leqq d^{\prime}$. But regarding $E$ as formed by superimposing $D$ on $\Delta$, we have since $f \geqq 0$.

$$
\bar{S}_{E} \leqq \bar{S}_{\Delta}
$$

Thus

$$
\bar{S} \leqq \bar{S}_{\mathrm{D}}<\bar{S}_{\Delta}+\frac{\epsilon}{2}<\bar{S}+\epsilon
$$

which proves (1).

In the same way we establish:

Let $f\left(x_{1} \cdots x_{m}\right) \leqq 0 . \quad$ Let

$$
\underline{S}=\max \underline{S}_{D}
$$

with respect to all rectangular divisions $D$. Then

$$
\lim _{d=0} \underline{S}_{D}=\underline{S}
$$


Theorem 2. Let $f\left(x_{1}, \cdots, x_{m}\right)$ be defined over the field $\mathfrak{A}$. Then the limits

$$
\lim _{d=0} \underline{S}_{D}, \quad \lim _{d=0} \bar{S}_{D}
$$

exist and are finite. They are called respectively the lower and upper integrals of $f$ over $\mathfrak{A}$ and are denoted by

$$
\int_{\mathfrak{N}} f d \mathfrak{A}=\int_{\mathfrak{M}} f d x_{1} \cdots d x_{m} ; \quad \int_{\mathfrak{x}} f d \mathfrak{A}=\int_{\mathfrak{M}} f d x_{1} \cdots d x_{m} .
$$

This is a corollary of theorem 1 . We have only to introduce an auxiliary function

$$
g\left(x_{1}, \cdots, x_{m}\right)=f\left(x_{1}, \cdots, x_{m}\right)+c
$$

and take the constant $c$ so that $g$ is either $\leqq 0$ or $\geqq 0$ in $\mathfrak{A}$ according as we wish to show the existence of the first or second of the above limits.

When

$$
\int_{\mathfrak{x}} f d \mathfrak{A}=\int_{\mathfrak{x}} f d \mathfrak{A}
$$

we say $f$ is integrable in $\mathfrak{A}$; the common value of these two limits we denote by

$$
\int_{\mathfrak{n}} f d \mathfrak{A}=\int_{\mathfrak{n}} f d x_{1} \cdots d x_{m}
$$

and call it the integral of $f$ over $\mathfrak{A}$.

We may now prove at once the following theorem:

TheOREM 3. Let $D$ be any rectangular division of norm $d$, and $\xi_{i}$ any point of $\mathfrak{A}$ in the cell $d_{i}$. Then if $f$ is integrable in $\mathfrak{A}$,

$$
\lim _{d=0} \sum f\left(\xi_{i}\right) d_{i}=\int_{\mathfrak{x}} f d \mathfrak{A}
$$

however the D's and ' 's are chosen. Conversely if this limit exists, $f$ is integrable in $\mathfrak{A}$.

It is now easy to show that the usual criteria for integrability hold for the present case.

An important class of integrable functions are those having limited variation. In fact, let $f\left(x_{1}, \cdots, x_{m}\right)$ be defined over $\mathfrak{A}$. Let $D$ be a cubical division of norm $d \leqq d_{0}$. If there exists a positive number $\omega$ such that

$$
\sum_{i} d^{m-1} \text { ose } f_{i}<\omega
$$


however $D$ be chosen, we say $f$ has limited variation in $\mathfrak{A}$; otherwise $f$ has unlimited variation.

TheоRem 4. If $f\left(x_{1}, \cdots, x_{m}\right)$ has limited variation in $\mathfrak{A}$, it is integrable in $\mathfrak{A}$.

For,

But

$$
\sum d_{i} \operatorname{osc} f_{i} \leqq \sum d^{m} \operatorname{osc} f_{i}<\omega d
$$

Hence $f$ is integrable.

$$
\lim _{d=0} \omega d=0 \text {. }
$$

\section{$\S 4$. Content of point aggregates.}

In all the following work the notion of content is fundamental. This has been defined in various ways, e. g., by Cantor, Bores, Jordan. The definition we adopt is equivalent to that of CANTOR and JORDAN, although on the face, it is different. We have chosen it because of its relation to the definition of a multiple integral just given.

Let $\mathfrak{A}$ be any limited point aggregate in $R_{m}$. Let us effect a division $D$ of space of norm $\delta$. Let

$$
d_{1}, d_{2}, d_{3}, \cdots
$$

denote those cells of $D$ containing at least one point of $\mathfrak{A}$, while

$$
d_{1}^{\prime}, d_{2}^{\prime}, d_{3}^{\prime}, \ldots
$$

denote those cells all of whose points lie in $\mathfrak{A}$.

Then the limits

exist and are finite.

$$
\overline{\mathfrak{A}}=\lim _{\delta=0} \sum d_{i} ; \quad \underline{\mathfrak{U}}=\lim _{\delta=0} \sum d_{i}^{\prime}
$$

For, let

$$
\begin{aligned}
g\left(x_{1}, \cdots, x_{m}\right) & =1 \text { at points of } \mathfrak{A} \\
& =0 \text { at other points. }
\end{aligned}
$$

Then obviously

$$
\overline{\mathfrak{A}}=\overline{\operatorname{cont}} \mathfrak{A}=\bar{\int}_{\mathfrak{i}} g d \mathfrak{A} ; \quad \underline{\mathfrak{A}}=\underline{\operatorname{cont}} \mathfrak{A}=\int_{\mathfrak{A}} g d \mathfrak{A} .
$$

The number $\overline{\mathfrak{A}}, \underline{\mathfrak{A}}$ are called the upper and lower content of $\mathfrak{A}$. When

$$
\overline{\mathfrak{A}}=\underline{\mathfrak{I}},
$$

their common value is called the content of $\mathfrak{A}$; in this case we say $\mathfrak{A}$ is measurable. 
We observe that criteria for the integrability of $f\left(x_{1}, \ldots, x_{m}\right)$ give at once criteria for the measurability of $\mathfrak{A}$. When no ambiguity can arise, we may denote the content of $\mathfrak{A}$ by the same letter, otherwise we denote it by

cont $\mathfrak{A}$.

When cont $\mathfrak{A}=0$, we say that $\mathfrak{A}$ is discrete. We show at once :

For $\mathfrak{A}$ to be measurable, it is necessary that the content of its frontier points $\mathfrak{F}$ be 0 ; i. e that $\mathfrak{F}$ be discrete.

With the points of an aggregate $\mathfrak{A}$ let us form partial aggregates $\mathfrak{A}_{1}, \ldots, \mathfrak{A}_{4}$, such that the aggregate of the common frontier points of any two of these aggregates is discrete. We shall say that we have divided $\mathfrak{A}$ into the unmixed aggregates $\mathfrak{A}_{1}, \ldots, \mathfrak{A}_{\text {, }}$.

Theorem 5. If we divide $\mathfrak{A}$ into the unmixed aggregates $\mathfrak{A}_{1}, \ldots, \mathfrak{A}_{\iota}$, then

$$
\overline{\mathfrak{A}}=\overline{\mathfrak{A}}_{1}+\cdots+\overline{\mathfrak{A}}_{\mathrm{s}} ; \quad \underline{\mathfrak{A}}=\underline{\mathfrak{A}}_{1}+\cdots+\underline{\mathfrak{A}}_{0} .
$$

For, let $D$ be a rectangular division of norm $\delta$. Let $\overline{\mathfrak{F}}_{D}$ be the volume of those cells of $D$ which contain points of more than one of the aggregates $\mathfrak{A}_{1}, \ldots, \mathfrak{A}_{i}$. Let $\overline{\mathfrak{A}}_{i, D}$ be the volume of those cells containing points of $\mathfrak{A}_{i}(i=1,2, \cdots, s)$. Then

$$
\overline{\mathfrak{A}}_{D} \leqq \overline{\mathfrak{A}}_{1, D}+\cdots+\overline{\mathfrak{A}}_{s, D} \leqq \overline{\mathfrak{A}}_{D}+s \overline{\mathfrak{F}}_{D} .
$$

By hypothesis

$$
\lim _{\delta=0} \overline{\mathfrak{F}}_{D}=0
$$

Hence passing to the limit in (1), we get the first half of the theorem. The other half follows similarly.

In showing that certain aggregates have content, the two following theorems are sometimes useful. They rest on the notion of a total difference quotient.

Let $f\left(x_{1}, \ldots, x_{m}\right)$ be defined over $\mathfrak{A}$. Let $x=\left(x_{1}, \therefore, x_{m}\right)$ be an arbitrary but fixed point of $\mathfrak{A}$ while $x^{\prime}=\left(x_{1}+h_{1}, \ldots, x_{m}+h_{m}\right)$ is a variable point of $\mathfrak{A}$. Let $\Delta f=f\left(x^{\prime}\right)-f(x)$, and $\Delta x=\operatorname{dist}\left(x, x^{\prime}\right)$. The quotient

$$
\frac{\Delta f}{\Delta x}
$$

may be called the total difference quotient of $f$ at $x$. The point $x^{\prime}$ may, or may not, be restricted to remain near $x$, as required.

Let

$$
y_{1}=f_{1}\left(x_{1}, \cdots, x_{m}\right), \cdots, y_{n}=f\left(x_{1}, \cdots, x_{m}\right)
$$

be defined over $\mathfrak{A} . \quad$ As $x=\left(x_{1}, \cdots, x_{m}\right)$ ranges over $\mathfrak{A}$, the point $y=\left(y_{1}, \cdots, y_{n}\right)$ 
will range over an aggregate $\mathfrak{Y}$ in an $n$-way space $R_{n}$. We call $\mathfrak{B}$ the image of $\mathfrak{A}$.

Theorem 6. If $\mathfrak{A}$ is discrete, and the functions

$$
y_{1}=f\left(x_{1}, \cdots, x_{m}\right), \cdots, y_{n}=f_{n}\left(x_{1}, \ldots, x_{m}\right) \quad(n=m+p \geqq m)
$$

have limited total difference quotients, the image $\mathfrak{B}$ of $\mathfrak{A}$ is discrete.

TrEorem 7. Let the functions

$$
y_{1}=f_{1}\left(x_{1}, \cdots, x_{m}\right), \cdots, y_{n}=f_{n}\left(x_{1}, \cdots, x_{m}\right) \quad(n=m+r>m)
$$

have limited total difference quotients in $\mathfrak{A}$ except at points of a discrete aggregate $\Delta$. In the cells of any cubical division of norm $d \leqq d_{0}$, let at least $m$ of these difference quotients remain limited. Then $\mathfrak{B}$, the image of $\mathfrak{A}$, is discrete.

We prove only theorem 6 , as the demonstration of 7 is similar. Let us effect a cubical division $D$ of the $x$-space of norm $d$. Since the difference quotients are limited,

$$
\left|\Delta f_{i}\right|<d G \quad(i=1,2, \cdots, m),
$$

when $x$ ranges over any one of the cells $d_{i}$ of $D$. Hence each coördinate remains in an interval of length $<d G$ as $x$ ranges over the points of $\mathfrak{A}$ in $d_{i}$. Hence $y=\left(y_{1}, \cdots, y_{n}\right)$ remains within a cube of volume $d^{n} G^{n}$, and thus

$$
\overline{\mathcal{B}}<\sum_{*} d^{n} G^{n}=d^{n} G^{n} \sum_{: 1} d^{m}=d^{p} G^{n} \overline{\mathfrak{A}}_{D} .
$$

As $\lim \overline{\mathfrak{A}}_{D}=0$, it follows that $\overline{\mathcal{W}}=0$; hence cont $\mathfrak{W}=0$.

It is convenient to extend the terms cells, division of space into cells, etc., as follows. Let us suppose the points of any aggregate $\mathfrak{A}$, which may be $R_{m}$, arranged in partial aggregates which we shall call cells and which have the following properties :

$1^{\circ}$. There are only a finite number of cells in any limited portion of space.

$2^{\circ}$. The frontier of each cell is discrete.

$3^{\circ}$. Each cell lies in a cube of side $\leqq \delta$.

We shall call this a division of $\mathfrak{A}$ of norm $\delta$.

Let $\Delta$ be such a division of space. As in the case of rectangular division, $\overline{\mathfrak{A}}_{\Delta}$ may denote the contents of all the cells which contain at least one point of $\mathfrak{A}$; while $\mathfrak{A}_{\Delta}$ may denote the content of those cells all of whose points lie in $\mathfrak{A}$.

It is now easy to show that

Theorem 8. $\quad \lim _{\delta=0} \overline{\mathfrak{A}}_{د}=\overline{\mathfrak{A}}, \quad \lim _{\delta=0} \mathfrak{\mathfrak { A }}_{\mathrm{J}_{\mathbf{S}}}=\underline{\mathfrak{A}}$.

If $\mathfrak{A}$ does not contain all of its limiting points, these may be adjoined to $\mathscr{A}$. The resulting aggregate is called the completed aggregate. 
Theorem 9. An aggregate $\mathfrak{A}$ and its completed aggregate $\mathscr{P}$ have the same upper content.

For, let us effect a rectangular division $D$ of norm $d$. The cells containing points of $\mathscr{V}$ fall into two classes: $1^{\circ}$ those cells $d_{1}, d_{2}, \ldots$ containing points of $\mathfrak{A} ; 2^{\circ}$ those cells $e_{1}, e_{2}, \ldots$ containing no point of $\mathfrak{A}$. Each of these latter cells as $e_{i}$ is contiguous to at least one cell $d_{k}$. If $e_{i}, \cdots$ are contiguous to $d_{k}$ we will join them to $d_{k}$ to form a new cell $\delta_{k}$, in such a way that each $e$-cell has been joined to some one $d$-cell. The cells $\delta_{1}, \delta_{2}, \ldots$ together with the cells $d_{1}, d_{2}, \ldots$ which remain unchanged by this process of consolidation define a division $\Delta$. The norm $\delta$ of this division converges to 0 with $d$.

But for the division $\Delta$,

and by theorem 8 ,

$$
\overline{\mathfrak{A}}_{\mathrm{\Delta}}=\overline{\mathrm{V}}_{\mathrm{s}} ;
$$

Hence

$$
\lim _{\delta=0} \overline{\mathfrak{A}}_{د}=\overline{\mathfrak{A}} \text {. }
$$

We may now show at once :

$$
\overline{\mathfrak{B}}=\overline{\mathfrak{A}} .
$$

ThEOREM 10. If $\mathfrak{A}$ is measurable, its completed aggregate is also measurable; and both have the same content.

Theorem 11. If $\mathfrak{A}$ is measurable, it has the same content as its derivative $\mathfrak{A}^{\prime}$.

Connected with any complete aggregate $\mathfrak{A}$ of upper content $\overline{\mathfrak{A}}>0$, is an aggregate $\mathfrak{B}$, obtained from $\mathfrak{A}$ by a process of sifting, and therefore it may be called the sifted aggregate of $\mathfrak{A}$.

Let $D_{1}, D_{2}, \ldots$ be a set of rectangular divisions of space each formed by superimposing a division on the preceding. Let the norms of these divisions converge to zero. The division $D_{1}$ effects a division of $\mathfrak{A}$ into unmixed partial aggregates. Let $\mathfrak{A}_{1}$ denote those partial aggregates whose upper content is $>0$. Then $\overline{\mathfrak{A}}_{1}=\mathfrak{A}$. Similarly $D_{2}$ defines a partial aggregate of $\mathfrak{A}_{1}$, such that $\overline{\mathfrak{A}}_{2}=\mathfrak{A}$. As this process goes on, the cells of $D_{n}$ containing points of $\mathfrak{U}_{n}$ diminish in size and, for $n=\infty$, converge to a set of points $\mathfrak{B}$. The upper content of the points of $\mathfrak{A}$ in the domain of any point of $\mathfrak{B}$ is $>0$. Thus each point of $\mathfrak{Y}$ is a limiting point of $\mathfrak{A}$, and hence is a point of $\mathfrak{A}$. It is now easy to show that $\mathfrak{B}$ contains no isolated points, and is hence perfect. Moreover

$$
\overline{\mathfrak{Z}}=\overline{\mathfrak{A}} \text {. }
$$

\section{§5. Some properties of multiple integrals.}

Theorem 12. Let $f\left(x_{1}, \cdots, x_{m}\right)$ be defined over the field $\mathfrak{A}$. Let $\Delta$ be any division of norm $\delta$ into cells $\delta_{1}, \delta_{2}, \ldots$, not necessarily rectangular. Let $\mathfrak{M}_{i}, \mathfrak{m}_{i}$ be respectively the maximum and minimum of $f$ in $\delta$. Then 


$$
\begin{aligned}
& \lim _{\delta=0} \bar{S}_{\Delta}=\lim \sum \mathfrak{M}_{i} \delta_{i}=\int_{\mathfrak{q}}^{\bar{y}} f d \mathfrak{A} . \\
& \lim _{\delta=0} \underline{S}_{\Delta}=\lim \sum \mathfrak{m}_{i} \delta_{i}=\int_{\mathfrak{X}} f d \mathfrak{A} .
\end{aligned}
$$

For, let $D$ be a cubical division of norm $d$. Let $d_{1}, d_{2}, \ldots$ be the cells of $D$ containing points of $\mathfrak{A}$; while $M_{i}, m_{i}$ denote as usual the maximum and minimum of $f$ in $d_{i}$. Let $|f| \leqq F$ in $\mathfrak{A}$. For convenience, we also take $F \leqq 1$. Then for each $\varepsilon>0$ there exists a $d$ such that

$$
\left|\bar{S}_{D}-\overline{\int_{\mathfrak{a}}}\right|<\overline{\frac{\epsilon}{2}}
$$

We may also take $d$ so small that

$$
\overline{\mathfrak{A}}_{D}-\overline{\mathfrak{A}}<\frac{\epsilon}{8 F} .
$$

Consider now the division $\Delta$. Those of its cells containing points of $\mathfrak{A}$ fall into two classes: $1^{\circ}$ those lying in only one cell of $D ; 2^{\circ}$ those lying in more than one cell of $D$. Let $\delta_{i 1}, \delta_{i 2}, \ldots$ be the cells of the $1^{\circ}$ class lying in $d_{i}$; let $\delta_{1}^{\prime}, \delta_{2}^{\prime}, \ldots$ denote all the cells of the $2^{\circ}$ class.

Then the content of all the cells of $\Delta$ containing points of $\mathfrak{A}$ is

$$
\overline{\mathfrak{A}}_{\Delta}=\sum \delta_{i k}+\sum \delta_{i}^{\prime} .
$$

But since the frontier of $\overline{\mathfrak{A}}_{D}$ is discrete, there exists a $\delta_{0}$ such that

$$
\sum \delta_{i}^{\prime}<\frac{\epsilon}{16 F},
$$

for any $\delta \leqq \delta_{0} . \quad$ As moreover $\overline{\mathfrak{A}}_{\Delta}$ converges to $\overline{\mathfrak{A}}$, we may also suppose $\delta_{0}$ small enough that

$$
\overline{\mathfrak{A}}_{\Delta}-\overline{\mathfrak{A}}<\frac{\epsilon}{16 F}, \quad \delta \leqq \delta_{0} .
$$

From (5), (6) and (7) we have

which with (4) gives finally

$$
\left|\sum \delta_{i k}-\overline{\mathfrak{A}}\right|<\frac{\epsilon}{8 F},
$$

$$
\left|\sum \delta_{i k}-\overline{\mathfrak{A}}_{D}\right|<\frac{\epsilon}{4 F}
$$

Let $\mathfrak{M}_{i k}, \mathfrak{M}_{i}^{\prime}$ be the maxima of $f$ in $\delta_{i k}, \delta_{i}^{\prime}$ respectively. Then

$$
\mathfrak{M}_{i k} \leqq M_{i}, \quad \mathfrak{M}_{i} \leqq F .
$$


Hence

With (6) this gives

$$
\overline{S_{\perp}} \leqq \sum M_{i} \delta_{i k}+F \sum \delta_{i}^{\prime}
$$

$$
\left|\overline{\bar{S}}_{\Delta}-\sum M \Gamma_{i} \delta_{i k}\right|<\frac{\epsilon}{4} .
$$

But

$$
\begin{aligned}
\left|\bar{S}_{D}-\sum M I_{i} \delta_{i k}\right| & \leqq F\left(\sum l_{i}-\sum \delta_{i k}\right)=F\left(\mathfrak{I}_{1}-\sum \delta_{i k}\right) \\
& <\frac{\epsilon}{4}, \text { by }(8) .
\end{aligned}
$$

From (3), (9) and (10) we have

$$
\left|\bar{S}_{\Delta}-\bar{\int}_{n}\right|<\epsilon, \quad \delta \leqq \delta_{11}
$$

This proves (1); in a similar manner we may establish (2).

It is now easy to prove the following theorems :

Theorem 13. Let $f\left(x_{1}, \cdots, x_{m}\right)$ be defined over the measurable field $\mathfrak{A}$. Let $\Delta$ be an unmixed division of $\mathfrak{i l}$ of norm $\delta$. Let $\underline{S}$ be the maximum of $\underline{S}_{\Delta}$ and $\bar{S}$ be the minimum of $\overline{S_{د}}$, for all divisions $\Delta$ of norm $\delta \leqq \delta_{0}$. Then

$$
\underline{S}=\int_{\mathfrak{N}} f d \mathfrak{H}, \quad \bar{S}=\int_{: 1} f d \mathfrak{A} .
$$

Theorem 14. Let $f\left(x_{1}, \cdots, x_{m}\right)$ be integrable in $\mathcal{H} . \quad$ Let $\mathfrak{P}_{\eta}$ be a partial aggregate depending on $\eta>0$ such that $\overline{\mathcal{P}}_{\eta} \doteq \overline{\mathfrak{A}}$ as $\eta \doteq 0$. Then

$$
\lim _{\eta=1} \int_{\mathfrak{V}^{*}} f d \mathcal{H}=\int_{\mathfrak{\eta}} f d \mathfrak{A} \text {. }
$$

Theorem 15. Let $f\left(x_{1}, \ldots, x_{m}\right)$ be defined over $\mathcal{H}$. Let $\cdot \mathscr{H}^{\prime}$ be a partial aggregate of $\mathfrak{A}$ such that $\overline{\mathfrak{U}}=\overline{\mathrm{V}}$. Then

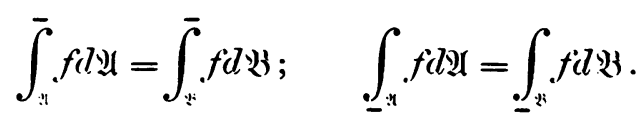

TheORem 16. Let $f\left(x_{1}, \ldots, x_{m}\right)$ be defined over the measurable field $\mathfrak{A}$, which is an inner field of $\mathscr{H}$. Let $g\left(x_{1}, \ldots, x_{m}\right)=0$ in $\mathcal{H}$, except at the points of $\mathfrak{A}$ where it $=f\left(x_{1}, \cdots, x_{m}\right)$. Then

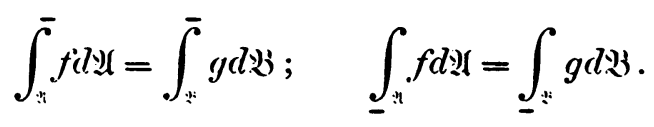


THEOnen 17. Let $f\left(x_{1}, \ldots, x_{m}\right)$ be defined over the field $\mathfrak{A}$ which is divided into the unmixed fields $\mathfrak{H}_{1}, \ldots, \mathfrak{H}_{s}$. Then

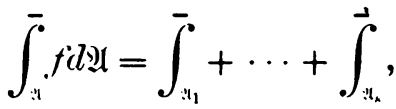

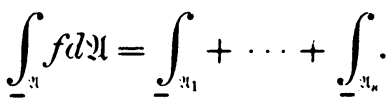

'Theorem 18. Let $f\left(x_{1}, \cdots, x_{m}\right) \geqq \lambda$ in $\mathfrak{A}$. Then

$$
\int_{\mathfrak{l}} f(\mathfrak{A} \geqq \lambda \overline{\mathfrak{A}} \text {. }
$$

THeоnem 19. Let $f\left(x_{1}, \cdots, x_{m}\right)$ be defined over the field $\mathfrak{A}$. Let $\mathfrak{A}_{\sigma}$ denote the points of $\mathfrak{A}$ at which $\left|f^{\prime}\right| \geqq \sigma . \quad$ If $\mathfrak{A}_{\sigma}$ is discrete for any $\sigma>0, f$ is integrable and

$$
\int_{\mathfrak{:}} f d \mathfrak{A}=0
$$

Thеонем 20. Let $f\left(x_{1}, \cdots, x_{m}\right)$ be integrable in the complete field $\mathfrak{A}$. Let $\mathfrak{C}$ be the points of $\mathfrak{A}$ at which $f$ is continuous. Then $\overline{\mathfrak{E}}=\overline{\mathfrak{A}}$.

For, if $\mathcal{U}$ is discrete, the theorem is true even if $f$ has no points of continuity in $\mathfrak{H}_{i}$. Let us therefore suppose that $\overline{\mathfrak{A}}>0$. Let $\mathfrak{B}$ be the sifted aggregate of $\mathfrak{A}$. Let $D$ be a rectangular division, and $d$ one of its cells containing points of $\mathfrak{B}$. We can choose $D$ so that no cell has points of $\mathfrak{B}$ only on its sides. Let $a$ be the points of $\mathcal{A}$ in $d$. Since $f$ is integrable in $\mathscr{A}$, it is in $a$. We can therefore choose a system of cubical subdivisions of a whose norms converge to zero, such that for each subdivision there is a cube lying in one of the preceding division, in which the oscillation is as small as we choose. These cubes converge to a point in $a$ at which $f$ is continuous. Returning now to the original division $D$, we see that every cell of $D$ which contains a point of $\mathfrak{B}$ contains a point of $\mathfrak{C}$. Hence $\overline{\mathbb{E}}_{D}=\overline{\mathfrak{V}}_{1}$; hence $\overline{\mathfrak{E}}=\overline{\mathfrak{Z}}=\overline{\mathfrak{A}}$.

THEOREM 21. Let $f\left(x_{1}, \cdots, x_{m}\right) \geqq 0$ in $\mathfrak{A} . \quad$ If

$$
\int_{i ! n} \operatorname{tin} \mathfrak{i}=0
$$

the points $\mathscr{H}_{\sigma}$ at which $f$ is equal to or greater than an arbitrarily small positive number $\sigma$, form a discrete aggregate. Let 3 denote the points at which $f=0 . \quad$ If $f^{\prime}$ is is complete, $\overline{\mathfrak{A}}=\overline{3}$.

For,

$$
0 \geqq \bar{\int}_{:_{\sigma}} \geqq \sigma \overline{\mathfrak{A}}_{\sigma} .
$$


Hence, $\overline{\mathfrak{A}}_{\sigma}=0$. To prove the second part of the theorem, let $b$ be a point of the sifted aggregate $\mathfrak{B}$ of $\mathfrak{A}$, at which $f$ is continuous. Then we can choose $\delta>0$ so small that $f \geqq \lambda>0$ in any $D_{\delta}(b)$. But the upper content of the points $\overline{\mathfrak{a}}$ of $\mathfrak{A}$ in $D(b)$ is $\overline{\mathfrak{a}}>0$. Hence

$$
\int_{\mathfrak{A}} f d \mathfrak{U} \geqq \int_{\mathfrak{a}} f d \mathfrak{a} \geqq \lambda \overline{\mathfrak{a}}>0 .
$$

Hence $f=0$ at every point of continuity of $\mathfrak{A}$ in $\mathfrak{B}$. Let now $D$ be a rectangular division of space. Any cell of $D$ which contains a point of $\mathfrak{B}$ also contains a point of continuity lying in $\mathfrak{B}$. Hence

which gives

$$
\overline{3}_{D} \geqq \mathfrak{B}_{D} ;
$$

$$
\overline{3} \geqq \overline{\mathfrak{B}}, \quad \text { or } \quad \overline{3}=\overline{\mathfrak{B}} \text {. }
$$

§ 6. Reduction of multiple integrals to iterated integrals.

Let $\mathfrak{A}$ be a point aggregate in $R_{m} . \quad$ As $x=\left(x_{1}, \cdots, x_{m}\right)$ ranges over $\mathfrak{A}, x_{i}$ will range over an aggregate $\mathfrak{x}_{i}$ on the $x_{i}$ axis which we call the projection of $\mathfrak{A}$ on this axis.

The points of $R_{m}$ for which one of the coördinates as $x_{i}$ has a fixed value $x_{i} \mp \xi_{i}$ lie in an $(m-1)$-way plane perpendicular to the $x_{i}$ axis. We denote it by $P_{\xi_{i}}$ or more shortly by $P_{i}$. The points of $\mathfrak{A}$ in $P_{\xi_{i}}$ form a plane section of $\mathfrak{A}$ corresponding to the point $\xi_{i}$ in $\mathfrak{x}_{i}$. We denote it by $\mathfrak{P}_{\xi_{i}}$ or by $\mathfrak{P}_{i}$.

As $x=\left(x_{1}, \cdots, x_{m}\right)$ ranges over $\mathfrak{A}$, the point $\left(x_{1}, \cdots, x_{i-1}, 0, x_{i+1}, \ldots, x_{m}\right)$ ranges over an aggregate $\mathfrak{X}_{i}$, called the projection of $\mathfrak{A}$ on the plane $x_{i}=0$.

Let us fix all the coördinates of $x=\left(x_{1}, \cdots, x_{m}\right)$ except $x_{i}$. Then $x$ describes a line; the points of $\mathfrak{A}$ on one of these lines we call the rectilinear section of $\mathfrak{A}$ parallel to the $x_{i}$ axis. We mention a few properties of these aggregates, whose demonstration is readily given.

TheOREM 22. If $\mathfrak{A}$ is complete, so are the aggregates $\mathfrak{x}_{i}, \mathfrak{X}_{i}, \mathfrak{a}_{i}, \mathfrak{P}_{i}$, above mentioned.

Theorem 23. Let $\mathfrak{2}$ be measurable. Let $\mathfrak{x}_{\sigma}$ denote those points of $\mathfrak{x}_{i}$ for which the upper content of the frontier points of $\mathfrak{P}_{i}$ is $\geqq \sigma$. Then $\mathfrak{x}_{\sigma}$ is dis. crete.

A similar theorem holds for the aggregate $\mathfrak{X}_{\sigma}$.

Theorem 24. Let $f\left(x_{1}, \cdots, x_{m}\right)$ be defined over the measurable field $\mathfrak{A}$. Then

$$
\begin{aligned}
& \int_{\mathfrak{A}} f d \mathfrak{A} \leqq \int_{\int_{\mathfrak{x}_{i}}} d x_{i}{\underline{\int_{\mathfrak{P}_{i}}}} f d \mathfrak{P}_{i} \leqq \bar{\int}_{\mathfrak{x}_{i}} d x_{i} \int_{\mathfrak{R}_{i}} f d \mathfrak{P}_{i} \leqq \int_{\mathfrak{B}} f d \mathfrak{A}, \\
& \int_{\mathfrak{A}} f d \mathfrak{U} \leqq \int_{\mathfrak{x}_{i}} d x_{i} \int_{\mathfrak{R}_{i}} f d \mathfrak{P} \leqq \int_{\mathfrak{x}_{i}} d x_{i} \int_{\mathfrak{*}_{i}} f d \mathfrak{P}_{i} \leqq \int_{\mathfrak{Q}} f d \mathfrak{A} \text {. }
\end{aligned}
$$


Let us prove (1); the demonstration of $(2)$ is similar. Let $\mathfrak{A}$ lie in an outer cube $\mathscr{V}$, whose projection on the $x_{i}$ axis is $\mathfrak{b}$, and whose plane sections perpendicular to this axis are $D$. We introduce the auxiliary function

$$
\begin{aligned}
g\left(x_{1}, \cdots, x_{m}\right) & =f\left(x_{1}, \cdots, x_{m}\right) \text { in } \mathfrak{A}, \\
& =0 \text { at other points of } \mathfrak{B} .
\end{aligned}
$$

Let $D$ be a cubical division of $R_{n}$ of norm $d$. This divides $B$ into cells which we denote by $\delta$. It also divides the plane section $\mathfrak{Q}$ into cells $\delta^{\prime}$, and the segment $\mathfrak{b}$ into intervals which we denote by $\delta^{\prime \prime}$.

Let $M, M^{\prime}$ be the maxima, and $m, m^{\prime}$ the minima of $g$ in the cells $\delta, \delta^{\prime}$. Let $|f| \leqq F$ in $\mathfrak{A}$. Let $\bar{G}, \underline{G}$ denote the upper and lower integrals of $g$ in the field $\mathscr{Y}$. Then for each $\epsilon>0$ there exists a $d_{0}$ such that

$$
\underline{G}-\epsilon<\sum_{\sharp} m \delta \leqq \sum_{\sharp} M \delta<\bar{G}+\epsilon
$$

Also for each $x_{i}$ of $\mathfrak{b}$

$$
\sum_{\Xi} n^{\prime} \delta^{\prime} \leqq \int_{\Xi} g d \rrbracket \leqq \sum_{\Omega} M^{\prime} \delta^{\prime} ;
$$

or since $m \leqq m^{\prime}$, and $M^{\prime} \leqq M$,

$$
\sum_{\Omega} m \delta^{\prime} \leqq \int_{\square} g d \emptyset \leqq \sum_{\Xi} M \delta^{\prime}
$$

Multiplying by $\delta^{\prime \prime}$ and summing over $\mathfrak{b}$ we have, since $\delta=\delta^{\prime} \delta^{\prime \prime}$,

$$
\sum_{*} m \delta \leqq \sum_{v} \delta^{\prime} \int_{D} \leqq \sum_{*} M \delta .
$$

Combined with (3), this gives

or

$$
\underline{G}-\epsilon<\sum_{\mathrm{b}} \delta^{\prime \prime} \int_{\Omega}<\bar{G}+\epsilon
$$

$$
\underline{G} \leqq \int_{1} \int_{\Omega} \leqq \int_{\mathfrak{b}} \int_{0} \leqq \bar{G}
$$

From (4) and theorem 16 we have

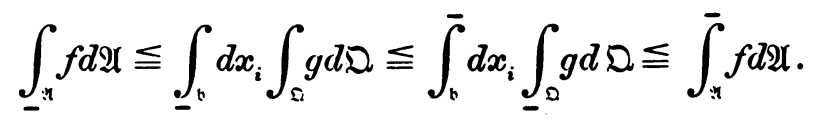

Let $\overline{\widetilde{F}}_{i}$ denote the upper content of the frontier points of $\mathfrak{P}_{i}$. Then

$$
\left|\int_{\mathbb{V}_{i}} f d \mathfrak{P}-\int_{\mathbb{D}} g d \mathfrak{D}\right| \leqq F \overline{\mathfrak{F}}_{\mathfrak{l}}
$$


Let us set

$$
\begin{aligned}
h\left(x_{i}\right) & =\int_{\mathfrak{P}_{i}} f d \mathfrak{P}_{i}, \text { for points of } \mathfrak{x}_{i} ; \\
& =0, \text { for other points of } \mathfrak{b} .
\end{aligned}
$$

Then by (6), since the points of $\mathfrak{x}_{i}$ for which $\overline{\mathfrak{F}}_{w}>\sigma$ form a discrete set,

But obviously

Hence from (7), (8),

$$
\int_{0} h d x_{i}=\int_{b} d x_{i} \int_{0} g d \Omega \text {. }
$$

$$
\int_{b} h d x_{i}=\int_{x_{i}} h d x_{i}
$$

$$
\int_{b} d x_{i} \int_{0} g d \mathbb{Q}=\int_{\mathcal{x}_{i}} d x_{i} \int_{\mathbb{k}_{i}} f d \mathfrak{F}_{i}
$$

In the same way we show

$$
\int_{b} d x_{i} \int_{0} g d \square=\int_{x_{i}} d x_{i} \int_{*} f d P_{i} .
$$

By placing (9) and (10) in (5) we get (1).

In a similar manner we may prove :

Theorem 25. Let $\mathfrak{A}$ be measurable. Let $\mathfrak{X}_{i}$ be its projection on the plane $x_{i}=0$. Let $\mathfrak{a}_{i}$ be the rectilinear section of $\mathfrak{A}$ parallel to the $x_{i}$ axis. Then

$$
\begin{aligned}
& \int_{\mathfrak{A}} f d \mathfrak{A} \leqq \int_{\mathfrak{x}_{i}} d \mathfrak{X}_{i} \int_{\mathfrak{u}_{i}} f d x_{i} \leqq \int_{x_{i}} d \mathfrak{X}_{i} \int_{\mathfrak{u}_{i}} f d x_{i} \leqq \int_{\mathfrak{A}}^{\bar{A}} f d \mathfrak{A} .
\end{aligned}
$$

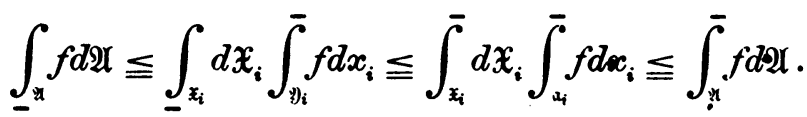

Many theorems follow now from 24 and 25 as corollaries. We state only the two following.

Theorem 26. Let $f\left(x_{1}, \cdots, x_{m}\right)$ be integrable in the measurable field $\mathfrak{A}$. Let $\mathfrak{x}_{\sigma}$ denote those points of $\mathfrak{x}_{i}$ for which

$$
\int_{\mathbb{R}_{i}} f d \mathfrak{\int _ { * }} f d \mathfrak{\sigma} \geqq
$$

Then $\mathfrak{x}_{\sigma}$ is discrete. If $\mathfrak{A}$ is complete, the upper content of the points at which the above difference vanishes is $\overline{\mathfrak{x}}_{i}$.

TheOREM 27. Let $f\left(x_{1}, \cdots, x_{m}\right)$ be integrable in the measurable complete field $\mathfrak{A}$. Let $\mathfrak{y}_{i}$ denote those points of $\mathfrak{x}_{i}$ for which the integrals over the cor- 
responding plane sections $\mathfrak{P}_{i}$ exist. Let $\mathfrak{Y}_{i}$ denote the points of $\mathfrak{X}_{i}$ for which the integrals over the corresponding rectilinear sections $\mathfrak{a}_{i}$ exist. Then

$$
\int_{\mathfrak{a}} f d \mathfrak{A}=\int_{\mathfrak{n}_{i}} d x_{i} \int_{\mathfrak{F}_{i}} f d \mathfrak{P}_{i}=\int_{\mathfrak{N}_{i}} d \mathfrak{Y}_{i} \int f d x_{i} .
$$

§ 7. Change of variables.

Let the transformation $T$ be defined by the equations

$$
T ; \quad x_{1}=\phi_{1}\left(t_{1}, \cdots, t_{m}\right), \cdots, x_{m}=\phi_{m}\left(t_{1}, \cdots, t_{m}\right),
$$

where the $\phi$ 's have continuous first derivatives in the region $R$. Let the correspondence between $R$ and its image $R_{T}$ be uniform. Let the determinant of the transformation $J \neq 0$. We say the transformation $T$ is regular.

It is easy to show now that to any inner aggregate $\mathfrak{A}$ in $R$ corresponds an inner aggregate $\mathfrak{A}_{T}$ in $R_{T}$. To inner and frontier points of $\mathfrak{A}$ correspond sim ilar points in $\mathfrak{A}_{T}$, and conversely.

As a direct consequence of theorem 6 we have

Theonem 28. If either $\mathfrak{A}$ or $\mathfrak{A}_{T}$ is measurable, so is the other. Also if one is discrete, so is the other.

We show next how the transformation $T$ can be expressed as the product of two simpler régular transformations.

Since $J \neq 0$, not all the first partial derivatives vanish at any point of $R . \bullet$ Let

$$
\frac{\partial \phi_{m}}{\partial t_{m}} \neq 0
$$

at a point $t$ and hence in a certain domain $D_{\delta}(t)$ of $t$. Let us define the first of these new transformations by

$$
T_{1} ; \quad u_{1}=t_{1}, \ldots, u_{m-1}=t_{m-1}, \quad u_{m}=\phi_{m}\left(t_{1}, \ldots, t_{m}\right) .
$$

Since $T_{1}$ is regular, it can be inverted, giving

$$
t_{1}=u_{1}, \cdots, t_{m-1}=u_{m-1}, t_{m}=\theta\left(u_{1}, \cdots, u_{m}\right),
$$

where $\theta$ is a one-valued function having continuous first partial derivatives in a certain domain $D_{\eta}(u)$. If $\delta^{\prime} \leqq \delta$ is taken sufficiently small, the image $U$ of $D_{\delta^{\prime}}(t)$ lies in $D_{\eta}(u)$. The second transformation is

$T_{2} ; \quad x_{1}=\phi_{1}\left(u_{1}, \cdots, u_{m-1}, \theta\right), \ldots, x_{m-1}=\phi_{m-1}\left(u_{1}, \cdots, u_{m-1}, \theta\right), \quad x_{m}=u_{m}$,

and is defined over $U$.

Obviously now

$$
T=T_{1} I_{2}
$$


when $t$ ranges over $D_{\delta^{\prime}}(t)$, and the determinant $J_{2}$ of $T_{2}$ is $\neq 0$, since the determinants $J, J_{1}$ of $T$ and $T_{1}$ are $\neq 0$, by hypothesis.

Let $\mathfrak{A}$ be an inner aggregate of $R$. It is easy now to show that we can effect a cubical division of the $t$-space of norm $d$ such that for the points of $\mathfrak{A}$ in each cell $d_{k}$, there exist two transformations $T_{1}^{(k)}, T_{2}^{(k)}$ of the kind just considered such that

$$
I=T_{1}^{(k)} T_{2}^{(k)} \text {. }
$$

For we can take $d$ so small that not all the first partial derivatives vanish in any cell, since otherwise $J=0$ at some point in this cell. Next, making use of the uniform continuity of our functions, we show that the norms $\eta$ of the domains $D_{\eta}(u)$, and hence the norms $\delta^{\prime}$ of $D_{\delta^{\prime}}(t)$ considered above, do not sink below some positive number, as $t$ ranges over any inner region of $R$. Thus taking $d>0$ small enough, the relation (1) holds in each cell containing a point of $\mathfrak{A}$.

ThEOREM 29. Let

$$
T ; \quad x_{1}=\phi_{1}\left(t_{1}, \cdots, t_{m}\right), \cdots, x_{m}=\phi_{m}\left(t_{1}, \cdots, t_{m}\right)
$$

define a regular transformation of determinant $J$ in the region $R . \quad$ Let $\mathfrak{T}$ be any inner perfect measurable aggregate of $R$ and let $\mathfrak{X}$ be its image. Let $f\left(x_{1}, \ldots, x_{m}\right)$ be continuous in $\mathfrak{X}$. Then

$$
\int_{x} f\left(x_{1}, \cdots, x_{m}\right) d x_{1} \cdots d x_{m}=\int_{x}|J| f d t_{1} \cdots d t_{m} .
$$

For $m=1$, the theorem is obviously true. Let us therefore assume it correct for $m-1$, and show it is so for $m$. As just shown, we can effect a cubical division of the $t$-space such that the relation (1) holds in each cell containing points of $\mathfrak{I}$. But if (2) holds for each of these partial aggregates of $\mathfrak{T}$, it obviously holds for $\mathfrak{T}$. We may therefore assume without loss of generality that the relation (1) holds throughout $\mathfrak{I}$. Let $\mathfrak{u}$ be the image of $\mathfrak{X}$ in the $u$-space, while $\mathfrak{y}_{m}^{\prime}, \mathfrak{P}_{m}^{\prime}$ are the images of $\mathfrak{y}_{m}, \mathfrak{P}_{m}$. Then

$$
\begin{aligned}
\int_{x} f d x_{1} \cdots d x_{m} & =\int_{y_{m}} d x_{m} \int_{*_{m}} f d x_{1} \cdots d x_{m-1} \\
& =\int_{\mathfrak{y}_{m}^{\prime}} d u_{m} \int_{\mathfrak{*}_{m}^{\prime}}\left|J_{2}\right| f d u_{1} \cdots d u_{m-1} \\
& =\int_{\mathfrak{u}}\left|J_{2}\right| d u_{1} \cdots d u_{m} .
\end{aligned}
$$

The application of the transformation $T_{2}$ to the integral on the right gives similarly, 


$$
\int_{\mathfrak{u}}\left|J_{2}\right| d u_{1} \cdots d u_{m}=\int_{\mathfrak{x}}\left|J_{1}\right|\left|J_{2}\right| f d t_{1} \cdots d t_{m} .
$$

This and the preceding equation give (2).

As a corollary of the last theorem we have

THEOREM 30. Let $d_{k}$ denote the volume of one of the cells of a rectangular division $D$ of norm $d$ in the t-space. Let $\mathfrak{T}$ be any inner region of $R$ and $\mathfrak{X}$ its image. To the division $D$ correspond a division $\Delta$ of the x-space into cells of volume $\delta_{k}$. The ceils of $\Delta$ following within $\mathfrak{X}$ are unmixed, and

$$
\delta_{k}=|J| d_{k}+\epsilon_{k}, \quad t \text { in } d_{k},
$$

where $\left|\epsilon_{k}\right|<\epsilon$ uniformly, if $d$ is taken sufficiently sinall.

TheOREM 31. Let $T$ be a regular transformation of determinant $J$ defined over a region $R$. Let $\mathfrak{T}$ be an inner aggregate of $R$ and let $\mathfrak{X}$ be its image. Let $f\left(x_{1}, \cdots, x_{m}\right)$ be limited in $\mathfrak{T}$. Then

$$
\begin{aligned}
\int_{\mathfrak{x}} f\left(x_{1}, \cdots, x_{m}\right) d \mathfrak{X} & =\int_{\mathfrak{x}}|J| f d \mathfrak{T}, \\
\int_{\mathfrak{x}} f\left(x_{1}, \cdots, x_{m}\right) d \mathfrak{X} & =\int_{\underline{x}}|J| f d \mathfrak{I} .
\end{aligned}
$$

Let us prove (3); the demonstration of (4) is similar. To the end we effect a cubical division of the $t$-space of norm $d$. To it corresponds a division of $\mathfrak{X}$ into cells of norm $\delta$.

Let us consider the integral on the left of (3). Emplcying the usual notation, and letting $J_{i}$ denote the value of $J$ at some point of $d_{i}$, we have

Here

$$
\begin{aligned}
\sum M_{i} \delta_{i} & =\sum M_{i}\left(\left|J_{i}\right|+\epsilon_{i}\right) d_{i} \\
& =\sum M_{i}\left|J_{i}\right| d_{i}+\sum \epsilon_{i} M_{i} d_{i} .
\end{aligned}
$$

$$
\left|\sum \epsilon_{i} M_{i} d_{i}\right|<\epsilon F^{\prime} \overline{\text { cont }} \mathfrak{I}=\eta,
$$

which may be made as small as we choose, on taking $d$ sufficiently small.

Let us consider now the integral on the right of (3). In the cell $d_{i}$,

$$
\max J \cdot \min |J| \leqq \max \cdot f|J| \leqq \max f \cdot \max |J|,
$$

if $\max f$ is positive, while the signs are reversed if it is negative. Let us set

Then

$$
M_{i}^{\prime}=\max \cdot f|J|, \text { in } d_{i} .
$$

where $\left|\epsilon_{i}^{\prime}\right|<\epsilon$ uniformly.

$$
M_{i}^{\prime}=M_{i}\left(\left|J_{i}\right|+\epsilon_{i}^{\prime}\right),
$$


Thus

(7)

$$
\sum M_{i}^{\prime} d_{i}=\sum M_{i}|J| d_{i}+\sum \epsilon_{i}^{\prime} M_{i} d_{i}
$$

where, as in (6),

Thus (5) and (7) give

$$
\left|\sum \epsilon_{i}^{\prime} M_{i} d_{i}\right|<\eta .
$$

which proves (3).

$$
\left|\sum M_{i} \delta_{i}-\sum M_{i} d_{i}\right|<2 \eta
$$

OUter Island, May, 1905. 